\title{
Offering male endoscopists as decoy option to nudge disinclined women to have colorectal cancer screening
}

S.T. Stoffel1,2, R. Kerrison1, I. Vlaev3, C. von Wagner1

1 Research Department of Behavioural Science and Health, University College London, London, UK.

${ }_{2}$ Business School, University of Aberdeen, Aberdeen, UK

3 Warwick Business School, University of Warwick, Coventry, UK

Contact information:

Christian von Wagner, $\mathrm{PhD}$

Research Department of Behavioural Science and Health

University College London

1-19 Torrington Place, WC1E 6BT, UK

Tel :+44 (0)207679 1614

Email: c.wagner@ucl.ac.uk

Compliance with ethical standards

Funding: This study was funded by Cancer Research UK (grant number C1418/A14134*).

Conflict of interest. The authors declare that they have no conflict of interest.

Ethical approval: All procedures performed in studies involving human participants were in accordance with the ethical standards of the institutional and/or national research committee and with the 1964 Helsinki declaration and its later amendments or comparable ethical standards.

Informed consent. Informed consent was obtained from all individual participants included in the study.

Word count: 3,100

Title Page w/ALL Author Contact Info

Number of pages: 16

Number of figures: 0

Number of tables: 4

Supplementary files: Pages 7, Tables 2, Figures 5.

* Funding agreement ensured the authors' independence in designing the study, interpreting the data, writing, and publishing the report

\begin{abstract}
Background. Previous studies have shown that a large proportion of women invited for bowel cancer screening prefer endoscopists of the same gender. We tested whether women who are initially disinclined to undergo flexible sigmoidoscopy screening would be more willing to have the test with a female practitioner if they were also offered a decoy appointment with a male practitioner.
\end{abstract}

Methods. We conducted two online experiments with women aged 35-54, living in England, who did not intend to undergo flexible sigmoidoscopy screening. In both experiments, women were randomised to two conditions: (1) control (appointment with a female endoscopist) and (2) decoy 
(two appointments to choose from, one with a male endoscopist and one with a female endoscopist).

Experiment $1(\mathrm{~N}=302)$ verified the conditions for the decoy using a conventional intention scale, while experiment $2(\mathrm{~N}=300)$ tested how the presence of the decoy influences the likelihood of women choosing the appointment with the female practitioner in a discrete choice task.

Results. While experiment 1 showed that the presence of the decoy increased intentions to attend the appointment with the female practitioner $(\mathrm{p}=0.02)$, experiment 2 confirmed that women were more likely to choose the appointment with the female endoscopist if they were also offered the decoy $(\mathrm{p}<0.001)$. In both experiments, the presence of the decoy decreased perceived difficulty of the screening decision and cognitive effort required to make the decision.

Conclusion. Offering disinclined women a male practitioner increased intention to have the test with an endoscopist of the same gender. This suggests that male screening practitioners can be used as decoy options to increase the likelihood that women choose female practitioners and facilitate the screening decision. 


\section{Background}

2 It is well established that women have a strong preference for female healthcare professionals across a wide range of health settings, including: primary care, gynaecology and gastroenterology (Fennema et al., 1990; Garcia et al., 2003; Graddy 1990; Kerseen et al. 1997; Meenes et al., 2005; Plunkett et al., 2002). In the context of gastroenterology, specifically, several studies have shown that, not only do a significant proportion of women have a preference for a female endoscopist, but that many would be willing to wait longer for an appointment in order to receive their preference, and some would even be more willing to undergo endoscopy if they were guaranteed their preferred practitioner gender (Fidler, et al. 2000; Menees et al. 2005; Stockwell et al. 2002; Varadarajulu et al. 2002; Farraye et al. 2004;

10 Stoffel et al., 2018).

To date, there is very little experimental evidence demonstrating that the offer of a same sex practitioner improves attendance at gastroenterology appointments. In a recent randomised online experimental survey, Stoffel and colleagues (2018) demonstrated that the offer of a same-sex practitioner was effective at improving intention among disinclined women approaching the eligible age for flexible sigmoidoscopy screening (commonly referred to as 'bowel scope screening' in England; Stoffel et al., 2018), although the effect was relatively small (improving intentions in only $17 \%$ of women). Furthermore, they did not find that offering women the possibility to choose the endoscopist's gender increased screening intentions over and above allocating women a female practitioner by default. They argued that this was caused by the strong preference for female endoscopists in their sample.

Behavioural economics, which analyses individual decision making by combining insights from economics and psychology, suggests that individual's preferences can be influenced by the presence of alternatives (Kahneman, 2011). Specifically, by offering an additional, less attractive, alternative (i.e. the 'decoy effect'; Huber et al., 1982). The fundamental idea underlying the decoy effect (the 'asymmetric-dominance' effect or 'attraction' effect) is that, given that human value judgments are relative and contextual (Vlaev et al., 2011), the additional, less attractive, alternative increases the perceived attractiveness of the target, thus increasing its likelihood of it being chosen. 
According to Thaler and Sustein, the decoy effect is a nudge technique that is aimed at influencing behaviour in a predictable way without forbidding any options or significantly changing their economic incentives (Thaler \& Sunstein, 2008). The effectiveness of the decoy effect has been consistently shown in the context of product selection (Huber et al., 1982; Doyle et al., 1999; SellersRubio \& Nicolau-Gonzalbez, 2015), gambling (Herne, 1999; Huber et al., 1982), employee selection (Tversky et al.,1988; Highhouse, 1996) and political elections (Herne, 1997; Pan et al., 1995).

To date, only a few studies have tested the decoy effect in the context of medical decision making, mainly because of ethical reasons (Rubaltelli et al., 2008; Schwartz \& Chapman, 1999; Stoffel et al., 2019; Zenko et al., 2016). As the principle of employing the decoy effect is to guide people towards a target behaviour, researchers must be careful that the target is desirable for both society and the individual. Medical treatments often bear benefits and potential harms which can be distressing for the individual. We are only aware of one study exploring whether the decoy effect could be applied to increase interest in CRC screening (Stoffel et al., 2019). The study showed that offering participants an inferior alternative screening appointment at a screening centre that was further away and/or required longer waiting times than the standard screening centre increased screening intentions for the standard appointment.

To test the hypothesis that male practitioners could be used as decoy alternatives, we performed two online experiments. Study 1 examined intention to undergo flexible sigmoidoscopy screening when offered an appointment with a female endoscopist or a choice of appointments with a male or female endoscopist. Study 2 investigated whether the alternative appointment offer of a male practitioner increased the likelihood of the female appointment being chosen in a discrete choice task. The aim of the first online experiment was to verify the conditions for the decoy (i.e. to confirm that disinclined women state lower intention to undergo flexible sigmoidoscopy with a male than a female practitioner), while the aim of the second experiment was to test whether the decoy increased the likelihood of the target being chosen. number 13439/005). 


\section{Experiment 1 - Examining intention as outcome}

56 Experiment 1 aimed to investigate whether the offer of a screening appointment with a male practitioner would serve as a decoy option. Specifically, experiment 1 examined: 1) whether women would state lower intention to attend the 'decoy' than the 'target' and; 2) whether women would be more likely to intend to attend the 'target' in the presence of the 'decoy'.

\section{Methods}

Like Stoffel and colleagues (2018), we recruited women aged 35-54 (living in England), without a prior bowel cancer diagnosis or removal of part of the bowel $(\mathrm{N}=1,216)$, from an online survey panel (Norstat; see S1 Figure in supplementary files). Participants received a small financial incentive from the survey vendor, which was defined by the length of the questionnaire, for completing the survey (around $£ 0.50$ ). Furthermore, we followed their approach to present eligible participants with a short description of flexible sigmoidoscopy, which is offered in England at no cost to men and women aged 55, and then asked them to correctly identify where the scope is inserted (to ensure they had read and understood the description of the screening test). Participants were not able to continue with the survey unless they could correctly answer the comprehension question (Stoffel et al., 2018; von Wagner et al., 2019). Participants were then asked to indicate their intention to undergo flexible sigmoidoscopy screening using the question: 'Would you take up the offer if you were invited to have the bowel scope screening test?' with responses on a fully labelled four-point scale ('definitely not', 'probably not', 'yes, probably' and 'yes, definitely'). Only 309 women (27.7\%) indicated that they would 'definitely not' $(\mathrm{N}=56)$ or 'probably not' $(\mathrm{N}=219)$ do the test and were therefore considered 'disinclined' and randomised to one of the two experimental conditions with equal probability. We chose to test the decoy among disinclined people to minimise ceiling and social desirability effects often associated with self-reported intention measures (Michie \& Abraham, 2004) and to simulate a targeted intervention aimed at non-attenders who are in most need of an effective behavioural intervention (Stoffel et al., 2018; Stoffel et al, 2019; von Wagner et al., 2019). Participants in both conditions were asked to imagine that they had received one, or, in the case of the decoy condition, two appointments for flexible sigmoidoscopy. In the control condition, the 
appointment offered was always with a female practitioner ('target'). In the intervention condition, one of the two appointments offered was with a female practitioner ('target'), while the other was with a male practitioner ('decoy'). ${ }^{1}$ To adjust for any confounding resulting from the time and the day of the appointment offered, participants were offered one (control) or two (intervention) of six possible appointment times / days using pseudorandom selection (the randomisation was designed to prevent the same time and day from being offered for both the male and female appointments presented to intervention participants).

\section{Measures}

Intention. We measured post-exposure intentions to attend the offered screening appointments with the question 'How likely would you be to attend appointment 1(2)?' using a slider ranging from 0 to 100 , where 0 indicates that they would definitely not attend the appointment, and 100 indicates that they would definitely attend the appointment.

Perceived decision difficulty and decision effort. In line with previous studies examining the decoy effect, we asked women in both conditions to state their perceived difficulty to state the intention on a fully labelled five-point Likert scale ('Not at all', 'Slightly', 'Moderately', 'Very' and 'Extremely') in response to the question 'How difficult was it for you to state your intention to attend the appointment(s)?' and to indicate their decision effort on a similar fully labelled five-point Likert scale ('None', 'Little', ‘Some', 'Considerate', and 'Great') for the question 'How much effort did you put into stating your intention to attend the appointment(s)?' Both difficulty and effort questions were adapted and simplified from a 12-item subjective measurement of mental load and mental effort (Krell \& Hui, 2017).

Cancer literacy and numeracy skills. At the end of the survey, responders' numeracy skill was assessed by the question 'Which of the following numbers represents the biggest risk of getting a disease?' with answer options ' $1 / 10$ ', '1/100', '1/1000' and 'I don't know', adapted from Lipkus and

\footnotetext{
${ }^{1}$ Our experimental conditions differed from Stoffel and colleagues' design (2018) in that we offered women specific appointments with day and time.
} 
colleagues (2001). Similarly, we measured cancer health literacy through six questions from Dumenci

107 and colleagues' CHLT-6 questionnaire (2014).

\section{$108 \quad$ Statistical analysis}

109 Due to the non-normal distribution of the intention answers (see S2 Figure in supplementary files), we used medians as measures of central tendency. We calculated confidence intervals for intention using nonparametric bootstraps. We used a Two-sample Wilcoxon rank-sum (Mann-Whitney) test to compare intention to attend the decoy appointment with intention to attend the target appointment for women in the decoy condition and a Wilcoxon-Mann-Whitney test to compare intention to attend the target across the two experimental conditions. Furthermore, we used unadjusted and adjusted ordered logistic regressions to investigate the effect of including the appointment with the male endoscopist on perceived decision difficulty and decision effort. The distribution of these two outcome variables are presented in S3 Figure in the supplementary files.

\section{Results}

119 The final sample consisted of 302 women, who were on average 42 years old, were mostly married or cohabiting $(\mathrm{N}=187 ; 61.9 \%)$, White-British $(\mathrm{N}=245 ; 81.1 \%)$, had at least A-levels $(\mathrm{N}=130 ; 57.0 \%)$, had paid work $(\mathrm{N}=187 ; 61.9 \%)$, and stated in the first intention question $(\mathrm{N}=233 ; 77.7 \%)$ that they would probably not undergo screening. S1 Table in the supplementary files shows that that there were no imbalances in sociodemographic characteristics among the two experimental conditions.

Table 1 shows that women in the decoy condition stated significantly lower intention to attend the decoy than the target (Median 10 vs 40, $\mathrm{p}<0.0001$ ). Similarly, women in the decoy condition stated significantly higher intention to attend the target appointment than those in the control condition (Median 40 vs 28.5, $\mathrm{p}<0.0181$ ).

$$
\text { < Table } 1 \text { about here > }
$$

The ordered logistic regression models presented in Table 2 show that women in the decoy group perceived the screening decision as less difficult (adjusted Odds Ratio (aOR): 0.60, 95\% Confidence 
Interval (95\% CI): $0.43-0.84, \mathrm{p}<0.01)$ and less effortful (aOR 0.48, 95\% CI: $0.34-0.66, \mathrm{p}<0.01)$ than women in the control group (see S3 Figure in the supplementary files).

Results of the first experiment confirmed that the additional offer of a screening appointment with a male practitioner increases intention to attend the appointment with a female practitioner. Thus, the male appointment offer seemingly acts as a decoy to the standard appointment by making the screening decision simpler as supported by our analysis investigating cognitive effort. However, our experiment examined intention and not choice between alternatives. Women in the decoy condition could indicate the same intention for both appointments. In fact, 34 women $(23.0 \%)$ indicated that they would be as likely to attend the appointment with the male practitioner as attend the appointment with the female practitioner. In experiment 2, we addressed the limitations of asking intention, by using a choice question to measure the decoy effect.

\section{Experiment 2 - Examining choice as outcome}

144 Experiment 2 tested whether a screening appointment with a male practitioner could be used as a decoy to increase the likelihood that women chose to have flexible sigmoidoscopy with a female endoscopist in a discrete choice task. Specifically, we asked women to state whether they would choose to attend one of the offered screening appointments or prefer to not attend either.

\section{Methods}

149 Our recruitment method for experiment 2 followed the first experiment. We recruited 1,130 women aged 35-54 (living in England), who were without prior bowel cancer diagnosis or removal of part of their bowel and didn't participate in the first experiment (Norstat; see S4 Figure in supplementary files). After excluding those who stated that they would undergo the flexible sigmoidoscopy (i.e. those who were not 'disinclined'), we ended up with a final sample of 300 disinclined women who were randomly assigned to the control $(\mathrm{N}=154)$ or the decoy condition $(\mathrm{N}=146)$. were told that they have received an appointment with a screening appointment with an endoscopist of 
the same gender; women in the decoy condition were offered two different appointments, one with a practitioner of the same gender and one with an endoscopist of the opposite gender.

159

\section{Measures}

Choice. In the control condition, respondents were asked to indicate their choice by responding to the question 'What would you choose?' with possible response options 'I would attend the appointment' and 'I would not attend the appointment' in the case of the control condition. Conversely, in the decoy condition, respondents were asked to choose between 'I would attend appointment 1, 'I would attend appointment 2' and 'I would not attend either of these appointments'.

Perceived decision difficulty and decision effort. Similar to experiment 1, we measured perceived decision difficulty with the question 'How difficult was it for you to answer whether you would confirm the (one of the two) appointment(s)?' Decisional effort was measured with the question 'How much effort did you put into deciding whether you would confirm the (one of the two) appointment(s)?' Response options to both questions were identical to experiment 1 .

Cancer literacy and numeracy skills. Both cancer literacy and numeracy skills were measured in the same way as in experiment 1 .

\section{Statistical analysis}

We used unadjusted and adjusted logistic regressions to investigate the effect of including the appointment with the male practitioner in the choice set on the frequency of women choosing the appointment with the female endoscopist. Similar to experiment 1, we use used unadjusted and adjusted ordered logistic regressions for perceived decision difficulty and decision effort. The distribution of these two outcome variables are presented in Supplementary Figure 5.

\section{Results}

Sociodemographic characteristics of the final sample were similar to experiment 1 , in that the average age was 43 years, most were married or cohabiting $(\mathrm{N}=183 ; 61.0 \%)$, White-British $(\mathrm{N}=255 ; 85.0 \%)$, had at least A-levels $(\mathrm{N}=188 ; 62.7 \%)$, had paid work $(\mathrm{N}=190 ; 63.3 \%)$, and stated in the first intention question ( $\mathrm{N}=219 ; 79.6 \%)$ that they would probably not undergo a flexible sigmoidoscopy. 
Importantly, there were no statistically significant differences in sociodemographic characteristics and initial intention (see S2 Table in supplementary files).

Table 3 shows that, in the unadjusted logistic regression, women were more likely to choose the appointment with the female practitioner if it was offered together with the appointment with the male practitioner (49.3\% vs $25.3 \%$, OR 2.87, 95\% CI: $1.76-4.67, p<0.001){ }^{2}$ This effect remained statistically significant after adjusting for covariates, including: initial intention, sociodemographic variables, own perceived bowel cancer risk, cancer literacy score and numeracy (aOR) 2.62, 95\% CI: $1.57-4.37, \mathrm{p}<0.01)$.

Similar to experiment 1 , Table 4 shows that responders in the decoy condition found the choice scenario less difficult than those in the control condition (aOR 0.63, 95\% CI $0.41-0.98, \mathrm{p}<0.05)$ and that they spent less cognitive effort in making the decision (aOR 0.62, 95\%CI: $0.41-0.95, \mathrm{p}<0.05$; see 53 Figure in the supplementary files).

\section{Discussion}

This study investigated whether one could increase intention to attend flexible sigmoidoscopy screening intentions, among disinclined women, by offering them additional appointments with male screening practitioners. Experiment 1 found that including appointments with male screening practitioners in the choice set increased intention to have the test with a female endoscopist. Experiment 2 replicated this finding when looking at choice instead of intentions. Here, women were more likely to choose the appointment with the female endoscopist when it was offered alongside an alternative appointment with a male practitioner. These results demonstrate that male screening practitioners can be used as decoy options to increase the likelihood that women choose female practitioners and facilitate the screening decision. It should be noted that while our results seem to

\footnotetext{
${ }^{2}$ Note that in line with Stoffel et al. 2019, we classified the 5 women in the decoy condition (3.4\%) who chose appointment with the male endoscopist as not wanting to attend the appointment with the female practitioner.
} 
contradict Stoffel and colleagues (2018) findings (they did not find any evidence that offering an active choice between male and female endoscopists would increase screening intention over and above allocating women female practitioners by default), we used a slightly different decision setting, which featured appointment dates and times, as well as different outcome variables (intention from 0 to 100 and discrete choice vs intention on a fully labelled four-point scale).

212 The results provide support that techniques from behavioural economics, such as nudge, can influence health behaviours (Marteau et al., 2012; Vlaev et al., 2016). Hollands and colleagues (2013) defined nudges as interventions that alter the properties or placement of objects or stimuli within microenvironments, with the goal to change behaviour. Nudges typically require minimal conscious engagement and can in principle influence the behaviour of many people simultaneously as they are not targeted to specific individuals. This definition focuses on the specific context including physical and social dimensions of micro-environments (e.g., spaces such as health centres). This definition also specifically reflects the focus on automatic processes that require minimal conscious engagement but does not exclude conscious and reflective processes. In contrast to nudge interventions, conventional public health tools (including cancer screening campaigns), usually include dissemination of information. An essential feature of those reflective strategies is their appeal to reflective mental processes to provoke informed choice. Our results reveal that this traditional approach can be enhanced by insights from behavioural economics.

Finally, this study had several limitations. It used hypothetical scenarios and non-representative online study samples. Furthermore, the experiments lacked behavioural validation, in that they only measured intentions and choice in a hypothetical setting. Thus, the next step would be to test the decoy offer of a male practitioner under more ecologically valid conditions with real behaviour in a randomised controlled trial.

\section{Open practices}

231 The materials and data for the experiments are available at OSF: 


\section{References}

234 Doyle, J. R., O'Connor, D. J., Reynolds, G. M., \& Bottomley, P. A. (1999). The robustness of the

235 asymmetrically dominated effect: Buying frames, phantom alternatives, and in-store purchases.

236 Psychology \& Marketing, 16(3), 225-243. https://doi.org/10.1002/(SICI)1520-

$237 \quad$ 6793(199905)16:3\%3C225::AID-MAR3\%3E3.0.CO;2-X

238 Dumenci, L., Matsuyama, R., Riddle, D.L., Cartwright, L.A., Perera, R.A., Chung, H., \& Siminoff,

239 L.A. (2014). Measurement of cancer health literacy and identification of patients with limited cancer

240 health literacy. J Health Commun, 19(Suppl 2), 205-24.

$241 \quad$ https://doi.org/10.1080/10810730.2014.943377

242 Farraye, F. A., Wong, M., Hurwitz, S., Puleo, E., Emmons, K., Wallace, M. B., \& Fletcher, R. H.

243 (2004). Barriers to endoscopic colorectal cancer screening: are women different from men?. The

244 American journal of gastroenterology, 99(2), 341. https://doi.org/10.1111/j.1572-0241.2004.04045.x

245 Fennema, K., Meyer, D. L., \& Owen, N. (1990). Sex of physician: patient's preferences and 246 stereotypes. Journal of Family Practice, 30(4), 441-447.

247 Fidler, H., Hartnett, A., Man, K. C., Derbyshire, I., \& Sheil, M. (2000). Sex and familiarity of 248 colonoscopists: patient preferences. Endoscopy, 32(06), 481-482. https://doi.org/10.1055/s-2000-645

249 Garcia, J. A., Paterniti, D. A., Romano, P. S., \& Kravitz, R. L. (2003). Patient preferences for 250 physician characteristics in university-based primary care clinics. Ethnicity and Disease, 13(2), 259251267.

252 Graffy, J. (1990). Patient choice in a practice with men and women general practitioners. Br J Gen 253 Pract, 40(330), 13-15.

254 Herne, K. (1997). Decoy alternatives in policy choices: Asymmetric domination and compromise 255 effects. European Journal of Political Economy, 13(3), 575-589. https://doi.org/10.1016/S0176$256 \quad \underline{2680(97) 00020-7}$ 
Herne, K. (1999). The effects of decoy gambles on individual choice. Experimental Economics, 2(1), 31-40. https://doi.org/10.1023/A:1009925731240

259

Highhouse, S. (1996). Context-dependent selection: The effects of decoy and phantom job candidates. https://doi.org/10.1006/obhd.1996.0006

Hollands, G. J., Shemilt, I., Marteau, T. M., Jebb, S. A., Kelly, M. P., Nakamura, R., Suhrcke, M., \& Ogilvie, D. (2013). Altering micro-environments to change population health behaviour: towards an evidence base for choice architecture interventions. BMC Public Health, 13, 1218. https://doi.org/10.1186/1471-2458-13-1218

Huber, J., Payne, J. W., \& Puto, C. (1982). Adding asymmetrically dominated alternatives: Violations of regularity and the similarity hypothesis. Journal of consumer research, 9(1), 90-98. https://doi.org/10.1086/208899

Kahneman, D. (2011). Thinking, fast and slow. New York: Farrar, Straus and Giroux.

270 Kerssens, J. J., Bensing, J., \& Andela, M. G. (1997). Patient preference for genders of health 271 professionals. Social science \& medicine, 44(10), 1531-1540. https://doi.org/10.1016/S0277$272 \quad \underline{9536(96) 00272-9}$

Krell, M., \& Hui, S.K.F. (2017). Evaluating an instrument to measure mental load and mental effort considering different sources of validity evidence, Cogent Education, 4:1. https://doi.org/10.1080/2331186X.2017.1280256

Lipkus, I.M., Samsa, G., Rimer, B.K. (2001). General performance on a numeracy scale among highly educated samples. Med Decis Making, 21(1), 37-44. https://doi.org/10.1177/0272989X0102100105 https://doi.org/10.1126/science.1226918 

https://doi.org/10.1016/S0016-5107(05)00540-7

Michie, S., \& Abraham, C. (2004). Interventions to change health behaviours: evidence-based or

Pan, Y., O'Curry, S., \& Pitts, R. (1995). The attraction effect and political choice in two elections. Journal of Consumer Psychology, 4(1), 85-101. https://doi.org/10.1207/s15327663jcp0401 04

Plunkett, B. A., Kohli, P., \& Milad, M. P. (2002). The importance of physician gender in the selection of an obstetrician or a gynecologist. American journal of obstetrics and gynecology, 186(5), 926-928. https://doi.org/10.1067/mob.2002.123401

Rubaltelli, E., Burra, P., Sartorato, V., Canova, D., Germani, G., Tomat, S., ... \& Rumiati, R. (2008). Strengthening acceptance for xenotransplantation: the case of attraction effect. Xenotransplantation, 15(3), 159-163. https://doi.org/10.1111/j.1399-3089.2008.00474.x

Schwartz, J. A., \& Chapman, G. B. (1999). Are more options always better? The attraction effect in physicians' decisions about medications. Medical Decision Making, 19(3), 315-323. https://doi.org/10.1177/0272989X9901900310

Sellers-Rubio, R., \& Nicolau-Gonzalbez, J. L. (2015). Testing the decoy effect in the presence of store brands. International Journal of Retail \& Distribution Management, 43(2), 113-125. https://doi.org/10.1108/IJRDM-07-2013-0144 Determinants of colorectal cancer screening in women undergoing mammography. The American journal of gastroenterology, 98(8), 1875-1880. https://doi.org/10.1111/j.1572-0241.2003.07577.x 
randomised experimental online survey. Journal of Medical Screening

308 Stoffel, S. T., Yang, J., Vlaev, I., \& von Wagner, C. (2019). Testing the decoy effect to increase

309 interest in colorectal cancer screening. PloS one, 14(3), e0213668,

310 https://doi.org/10.1371/journal.pone.0213668

311 Thaler, R. H., \& Sunstein, C. R. (2008). Nudge: Improving decisions about health, wealth, and

312 happiness. New Haven, CT: Yale University Press.

313 Tversky, A., Sattath, S., \& Slovic, P. (1988). Contingent weighting in judgment and choice.

314 Psychological Review, 95, 371-384. https://doi.org/10.1037/0033-295X.95.3.371

315 Varadarajulu, S., Petruff, C., \& Ramsey, W. H. (2002). Patient preferences for gender of endoscopists.

316 Gastrointestinal endoscopy, 56(2), 170-173. https://doi.org/10.1016/S0016-5107(02)70173-9

317 Vlaev, I., Chater, N., Stewart, N., \& Brown, G. D. A. (2011). Does the brain calculate value? Trends

318 in Cognitive Sciences, 15, 546-554. https://doi.org/10.1016/j.tics.2011.09.008

319 Vlaev, I., King, D., Dolan, P., \& Darzi, A. (2016). Theory and practice of 'nudging': Changing health

320 behaviors. Public Administration Review, 76, 550-561. https://doi.org/10.1111/puar.12564

321 von Wagner, C., Hirst, Y., Waller, J., Ghanouni, A., McGregor, L. M. Kerrison, R. S., ... \& Stoffel, S.

322 T. (2019). The impact of descriptive norms on motivation to participate in cancer screening -

323 Evidence from online experiments. Patient Education and Counseling. 0738-3991,

324 https://doi.org/10.1016/j.pec.2019.04.001

325 Zenko, Z., Ekkekakis, P., \& Kavetsos, G. (2016). Changing minds: Bounded rationality and heuristic

326 processes in exercise-related judgments and choices. Sport, Exercise, and Performance Psychology,

327 5(4), 337. https://doi.org/10.1037/spy0000069 